\title{
Application of Algebraic Combinatorics to Finite Spin Systems with Dihedral Symmetry
}

\author{
S. Bucikiewicz, L. DębSki and W. FloreK* \\ Institute of Physics, A. Mickiewicz University \\ Umultowska 85, 61-614 Poznań, Poland
}

(Received June 11, 2001; in final form July 13, 2001)

\begin{abstract}
Properties of a given symmetry group $G$ are very important in investigation of a physical system invariant under its action. In the case of finite spin systems (magnetic rings as $\mathrm{Fe}_{6}, \mathrm{Cu}_{6}, \mathrm{Fe}_{10}$, some planar macromolecules as $\mathrm{Fe}_{12}$ or $\mathrm{Fe}_{8}$ ) the symmetry group is isomorphic with the dihedral group $D_{N}$. In this paper group-theoretical "parameters" of such groups are determined, especially decompositions of transitive representations into irreducible ones and double cosets. These results are necessary to construct matrix elements of any operator commuting with $G$ in an efficient way. The approach proposed can be useful in many branches of physics, but here it is applied to finite spin systems, which serve as models for mesoscopic magnets.
\end{abstract}

PACS numbers: 02.20.Bb, 75.10.Jm, 75.75.+a

\section{Introduction}

One of standard ways of quantum magnets modeling is to consider a finite set of $N$ nodes carrying spins $s_{j}, 1 \leq j \leq N$, with a spin number $s$. At present such models are also used to investigate properties of magnetic macromolecules, e.g. $\left[\mathrm{NaFe}_{6}\left(\mathrm{OCH}_{3}\right)_{12}\left(\mathrm{C}_{17} \mathrm{H}_{15} \mathrm{O}_{4}\right)_{6}\right] \mathrm{ClO}_{4}\left(\mathrm{Fe}_{6}\right), \quad\left[\mathrm{Fe}_{10}\left(\mathrm{OCH}_{3}\right)_{20}\left(\mathrm{C}_{2} \mathrm{H}_{2} \mathrm{O}_{2} \mathrm{Cl}\right)_{10}\right]\left(\mathrm{Fe}_{10}\right)$, $\left[\left(\mathrm{PhSiO}_{2}\right)_{6} \mathrm{Cu}_{6}\left(\mathrm{O}_{2} \mathrm{SiPh}\right)_{6}\right] \cdot 6 \mathrm{EtOH}\left(\mathrm{Cu}_{6}\right),\left[\mathrm{Ni}_{12}\left(\mathrm{O}_{2} \mathrm{CMe}\right)_{12}(\mathrm{chp})_{12}\left(\mathrm{H}_{2} \mathrm{O}\right)(\mathrm{THF})_{6}\right]$ $\left(\mathrm{Ni}_{12}\right)$ or $\mathrm{Mn}_{12}$ acetate $[1]^{\dagger}$. The so-called Ising states or configurations, i.e. tensor products

* corresponding author; e-mail: Wojciech.Florek@spin.amu.edu.pl

$\dagger$ Since $\mathrm{Mn}_{12}$ acetate has tetragonal symmetry then the results obtained for the dihedral groups do not apply to this molecule. 


$$
\mu=\left|m_{1}, m_{2}, \ldots, m_{N}\right\rangle, \quad-s \leq m_{j} \leq s,
$$

of one-spin states $\left|m_{j}\right\rangle$ form a natural basis $\mathcal{B}$ of the quantum space of states $L$ with the dimension $\operatorname{dim} L=(2 s+1)^{N}$. In a general case, however, these states are not eigenvectors of the Heisenberg-like Hamiltonians. Therefore, it is necessary to solve an eigenproblem with $\operatorname{dim} L$ equations which, even using contemporary computers, is almost impossible for $N \approx 30$ and $s>1$. This is especially important when one wants to investigate spin correlations in the ground state and some of the first excited states of antiferromagnetic systems, since many methods allow us to determine eigenvalues, but it is much more difficult to find eigenvectors. Among others, it is caused by the numerical instability of necessary algorithms in the case of a very large number of linear equations [2]. The problem is partially solved by taking into account operators commuting with a given Hamiltonian: e.g. the $z$-th component of the total spin $S^{z}$ and irreducible representations of the Hamiltonian symmetry group [3, 4]. If the spin interactions are isotropic, then also the total spin number may be taken into account. However one has to find solutions of a set of homogeneous linear equations arising from the eigenproblem for $\boldsymbol{S}^{2}$ (in a finite spin system eigenvalues of $\boldsymbol{S}^{2}$ are known). In many interesting cases, for example, investigating the ground state of bipartite antiferromagnets, these solutions can be found exactly if one is able to cope with very large integers. Such tools are provided by multiple (or arbitrary) precision libraries like the GNU multiple precision arithmetic library (GMP or GNU MP in short) written and developed by T. Granlund [5]. However, such procedures work efficiently if the matrix of an operator (the squared total spin $\boldsymbol{S}^{2}$ or the Hamiltonian $\mathcal{H}$ in the presented considerations) is written in the symmetry adapted basis.

The considerations presented are illustrated by examples related with finite spin models, but a similar method can be applied in any case when:

- the space of states $L$ is spanned over orthonormal vectors (1), i.e. it is the $N$ th tensor power of the $n$-dimensional one-particle space;

- the considered operator $\mathcal{H}$, acting in $L$, commutes with a given permutation representation $P$ of a group $G$ (the Hamiltonian symmetry group).

Let a given group $G$ permutes vectors of a (finite) basis $\mathcal{B}$ of the quantum state space $L$. In the previous paper [6] general formulae for vectors of the irreducible basis and matrix elements of any operator commuting with all permutation operators $P(g), g \in G$, have been presented. The aim of the present work is to investigate the dihedral groups $D_{N}$ and obtain general analytical expressions in this case. These groups have been chosen, since they describe symmetry of molecular rings with $N$ identical spins $s$ [1]. In fact, these rings are also invariant under the reflection $\sigma_{h}$ in the horizontal plane, so the groups $D_{N h}$ should be considered. These groups are important for constructing symmetry coordinates of vibrating molecules or crystals, but in the case of spin models the action of $\sigma_{h}$ is trivial. Considering planar molecules one should introduce groups $C_{N v}$, which are elementary 
"bricks" for finite space groups [7]. However, groups $D_{N}$ and $C_{N v}$ are isomorphic and the abstract group $D_{N}$ is considered as a representative of isomorphic groups with the following generation relations [8]:

$$
D_{N}=\left\langle C_{N}, U_{0}\right\rangle, \quad C_{N}^{N}=U_{0}^{2}=\left(C_{N}^{j} U_{0}\right)^{2}=E, \quad 0 \leq j<N .
$$

The dihedral group $D_{N}$ consists of $2 N$ elements: $N$ powers of the $N$-fold rotation $C_{N}$ and perpendicular to it the twofold rotations $U_{j}=C_{N}^{j} U_{0}, 0 \leq j<N$. It is easy to write down general formulaefor multiplication (all calculations are done $\bmod N)$ :

$$
C_{N}^{j} C_{N}^{k}=C_{N}^{j+k}, \quad C_{N}^{j} U_{k}=U_{j+k}, \quad U_{j} C_{N}^{k}=U_{j-k}, \quad U_{j} U_{k}=C_{N}^{j-k} .
$$

The labeling scheme for $N=6$ is presented in Fig. 1 .

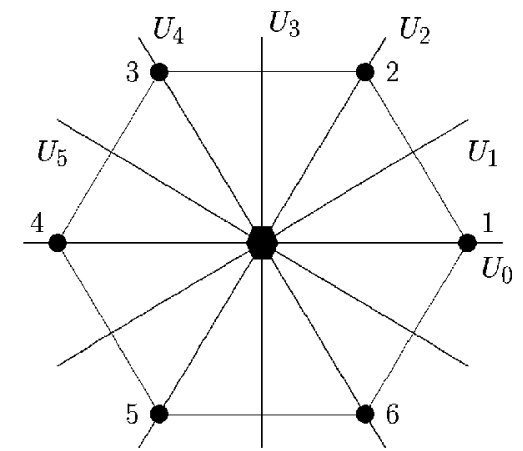

Fig. 1. Numbering of ring nodes and elements of the group $D_{6}$ (the sixfold axis $C_{6}$ is denoted by the full hexagon).

The dihedral groups are considered in Sec. 2 ; there are many differences between cases of $N$ odd and even, so the results are presented separately. In many actual applications one deals with bipartite antiferromagnets [1], so the case of $N$ even seems to be more important, however the other case ( $N$ odd) is discussed for three, at least, reasons: (i) the ground state energy of infinite linear antiferromagnets is approximated from below and above by those obtained in the cases of even- and odd-sized rings, respectively [4]; (ii) there are still some open problem related with linear ferromagnets (e.g. critical exponents near $T=0 \mathrm{~K}[9]$ ); in this case the parity of $N$ is (almost) irrelevant; (iii) the presented considerations and results are more complete. In Sec. 3 a partial solution for six spins $s=1$ (a ring invariant under $D_{6}$ ) is discussed. The paper ends with some conclusions.

\section{Dihedral groups $D_{N}$}

Let us recall the formulae obtained in the previous paper [6] to check which group properties should be determined. The irreducible basis consists of vectors $|U \mu ; \Gamma r \gamma\rangle$, where 
- $U \subseteq G$ is a subgroup of $G$ representing a conjugacy class $\widetilde{U}$;

- $\mu$ is a representative of an orbit of type $U$;

- $\Gamma$ is an irreducible representation (an irrep) of $G$, the repetition index $r$ distinguishes copies of $\Gamma$ and $\gamma=1,2, \ldots,[\Gamma]$ labels vectors of $\Gamma$.

The orbit $G(\mu)$ can be identified more precisely if one introduces a label denoting a non-ordered partition $[k]$ such that $\mu \in \mathcal{B}_{[k]}$. Note that $[k]$ identifies also the magnetization $M$. However, the formula for vectors of the irreducible basis does not depend on $[k]$ and is given as $[6]$

$$
|U \mu ; \Gamma r \gamma\rangle=\sum_{j=1}^{|G / U|} b_{j}^{\Gamma r \gamma}(U)\left|U \mu g_{j}\right\rangle,
$$

where the elements $g_{j}, 1 \leq j \leq|G / U|$ with $g_{1}=E$, represents the left cosets $g U \in G / U$. The coefficients $b_{j}^{\Gamma r \gamma}$, determining a unitary transformation, can be expressed by matrix elements of the irrep $\Gamma$ and the reduction coefficients $A_{\gamma r}^{U \Gamma}$ :

$$
b_{j}^{\Gamma r \gamma}(U)=\left(\frac{[\Gamma]|U|}{|G|}\right)^{1 / 2} \sum_{\beta=1}^{[\Gamma]} \Gamma_{\gamma \beta}^{*}\left(g_{j}\right)\left(A_{\beta r}^{U \Gamma}\right)^{*} .
$$

Matrix elements of any operator, e.g. a Hamiltonian $\mathcal{H}$, commuting with all $P(g)$, are grouped in blocks labeled by $\Gamma$ and depend only on $U, \mu$, and $r$ :

$$
h_{U \mu r, V \nu s}(\Gamma)=\left(\frac{|U|}{|V|}\right)^{1 / 2} \sum_{j=1}^{|G / U|}\left\langle U \mu g_{j}|\mathcal{H}| V \nu E\right\rangle B_{r s}^{U V \Gamma}\left(g_{j}\right),
$$

where

$$
B_{r s}^{U V \Gamma}\left(g_{j}\right)=\sum_{\alpha, \beta}\left(A_{\alpha s}^{V \Gamma}\right)^{*} \Gamma_{\alpha \beta}\left(g_{j}\right) A_{\beta r}^{U \Gamma} .
$$

It is helpful to consider non-ordered partitions $[k]$, because matrix elements are equal to zero if partitions $[k]$ and $\left[k^{\prime}\right]$ are not joined by an edge in the graph introduced in Sec. 5 of the previous paper [6]. Introducing the notion of double cosets $V g U$ this formula can be modified and written as

$$
h_{U \mu r, V \nu s}(\Gamma)=(|U||V|)^{-1 / 2} \sum_{d=1}^{|V \backslash G / U|}\left|V g_{d} U\right| B_{r s}^{U V \Gamma}\left(g_{d}\right)\left\langle U \mu g_{d}|\mathcal{H}| V \nu E\right\rangle
$$

where elements $g_{d}$ represent double cosets $V g_{d} U$, or orbits of the natural action ${ }_{V}(G / U)$.

All structures appearing in these formulae (cosets, irreps, reduction coefficients, etc.) in the case $G=D_{N}$ are discussed in the following subsections. 


\subsection{Classes and irreducible representations}

The multiplication rules (3) in the dihedral group $D_{N}$ lead immediately to classes of conjugated elements:

$$
\begin{aligned}
N \text { odd } & \{E\},\left\{C_{N}^{j}, C_{N}^{N-j}\right\}, \quad j=1,2, \ldots, \frac{N-1}{2}, \quad\left\{U_{0}, U_{1}, \ldots, U_{N-1}\right\} ; \\
N \text { even } & \{E\},\left\{C_{N}^{j}, C_{N}^{N-j}\right\}, \quad j=1,2, \ldots, \frac{N}{2}-1, \\
& \left\{C_{2}=C_{N}^{N / 2}\right\}, \quad\left\{U_{0}, U_{2}, \ldots, U_{N-2}\right\} \quad\left\{U_{1}, U_{3}, \ldots, U_{N-1}\right\} .
\end{aligned}
$$

Therefore, there are $(N+3) / 2$ classes (and irreps) for $N$ odd and $N / 2+3$ for $N$ even. In the special cases, $N=1,2$, the groups $D_{N}$ are Abelian.

In both cases, $N$ odd and even, there are two one-dimensional irreps, denoted $A_{1}$ (the unit irrep) and $A_{2}$, with characters

$$
\begin{aligned}
& \chi^{A_{1}}(g)=1, \quad \forall g \in D_{N} \\
& \chi^{A_{2}}\left(C_{N}^{j}\right)=1, \quad \chi^{A_{2}}\left(U_{j}\right)=-1, \quad j=0,1, \ldots, N-1 .
\end{aligned}
$$

For $N$ even there are two more one-dimensional irreps, denoted $B_{1}$ and $B_{2}$, with characters $(j=0,1, \ldots, N-1)$

$$
\begin{array}{ll}
\chi^{B_{1}}\left(C_{N}^{j}\right)=(-1)^{j}, & \chi^{B_{1}}\left(U_{j}\right)=(-1)^{j} ; \\
\chi^{B_{2}}\left(C_{N}^{j}\right)=(-1)^{j}, & \chi^{B_{2}}\left(U_{j}\right)=-(-1)^{j} .
\end{array}
$$

Note that the element $C_{2}=C_{N}^{N / 2}$ behaves in different ways for $N=4 M$ and $N=4 M+2$. In the first case one obtains $\chi^{B_{1}}\left(C_{2}\right)=\chi^{B_{2}}\left(C_{2}\right)=1$, whereas in the second case this character equals -1 .

The other irreps, denoted $E_{l}, l=1,2, \ldots, N_{E}$, are two-dimensional; there are $N_{E}=(N-1) / 2$ and $N_{E}=N / 2-1$ such irreps for $N$ odd and even, respectively. Their characters are given by the same formulae

$$
\chi^{E_{l}}\left(C_{N}^{j}\right)=2 \cos (2 \pi j l / N), \quad \chi^{E_{l}}\left(U^{j}\right)=0 .
$$

To complete the presentation, matrices of the representations $E_{l}$ in the standard basis $\{|E x\rangle,|E y\rangle\}$ for the generators $C_{N}$ and $U_{0}$ are written down:

$$
E_{l}\left(C_{N}\right)=\left(\begin{array}{rr}
\cos (2 \pi j l / N) & -\sin (2 \pi j l / N) \\
\sin (2 \pi j l / N) & \cos (2 \pi j l / N)
\end{array}\right), E_{l}\left(U_{0}\right)=\left(\begin{array}{rr}
1 & 0 \\
0 & -1
\end{array}\right)
$$

\subsection{Subgroups and conjugated subgroups}

Let $N$ be written as a product of $r$ different primes $p_{i}$ :

$$
N=\prod_{i=1}^{r} p_{i}^{\alpha_{i}}, \quad \alpha_{i}>0 .
$$

Then there are $\mathcal{D}=\prod_{i=1}^{r}\left(\alpha_{i}+1\right)$ different divisors $k$ of $N$, each of them can be written as $\left(k=1\right.$ corresponds to all $\left.\beta_{i}=0\right)$ 
$k=\prod_{i=1}^{r} p_{i}^{\beta_{i}}, \quad 0 \leq \beta_{i} \leq \alpha_{i}$.

The first family of subgroups consists of the cyclic groups $C_{k}$, where $k$ is a divisor of $N$. These groups are generated by elements $C_{N}^{\kappa}$, where $\kappa=N / k$ is the so-called co-divisor of $k$. For $k=1$ one obtains the group $C_{1}=\{E\}$, which has no generators; in this case $\kappa=N$. Each subgroup $C_{k}$ contains $k$ elements in the form $C_{N}^{p \kappa} \equiv C_{k}^{p}, p=0,1, \ldots, k-1$. Since these groups are normal divisors of $D_{N}$, then each $C_{k}$ is conjugated to itself only.

The second family of subgroups is formed by the dihedral groups $D_{k}$. However, for each $k$, i.e. for each generator $C_{N}^{\kappa}$, one has freedom in a choice of twofold generator $U_{j}$. The subgroups isomorphic to $D_{k}$ are generated by $C_{N}^{\kappa}$ and one of the rotations $U_{0}, U_{1}, \ldots, U_{\kappa-1}$. These subgroups will be denoted hereafter as

$$
D_{k}^{q}=\left\langle C_{N}^{\kappa}, U_{q}\right\rangle, \quad q=0,1, \ldots, \kappa-1 .
$$

Each of these groups, except for the rotations $C_{N}^{p \kappa}$, contains twofold axes $U_{q+p \kappa}, p=$ $0,1, \ldots, k-1$. In the case of odd $\kappa$ these elements belong to the same conjugacy class, so all subgroups $D_{k}^{q}$ are conjugated. This conjugacy class is represented by $D_{k}=\left\langle C_{N}^{\kappa}, U_{0}\right\rangle$. When $\kappa$ is even, then all twofold $U_{j}$ rotations in the subgroup $D_{k}^{q}$ have indices with the same parity. Therefore, there are two classes of conjugated subgroups: the first is represented by $D_{k}^{0}=\left\langle C_{N}^{\kappa}, U_{0}\right\rangle$ and the second by $D_{k}^{1}=\left\langle C_{N}^{\kappa}, U_{1}\right\rangle$. If $N$ is even then it can be written as $2^{\alpha} N^{\prime}$, where $N^{\prime}$ is odd. In the same way all divisors $k$ of $N$ can be written as $2^{\beta} k^{\prime}$. If $\beta=\alpha$ then corresponding $\kappa=N^{\prime} / k^{\prime}$ is odd and, therefore, there is only one class of conjugated subgroups $D_{k}$. Two separated classes, $D_{k}^{0}$ and $D_{k}^{1}$, are obtained in the other cases, i.e. when $\beta<\alpha$ and $\kappa=2^{\alpha-\beta} N^{\prime} / k^{\prime}$. Let $\mathcal{D}^{\prime}=\mathcal{D} /(\alpha+1)$ be the number of divisors of $N^{\prime}$ $\left(\mathcal{D}^{\prime}=\mathcal{D}\right.$ if $N$ is odd) then:

- there are $\mathcal{D}^{\prime}$ classes of dihedral subgroups in type $D_{k}$;

- in each type $D_{k}^{0}$ and $D_{k}^{1}$ there are $\alpha \mathcal{D}^{\prime}$ classes of dihedral subgroups.

Therefore, there are $(\alpha+1) \mathcal{D}^{\prime}$ cyclic subgroups and $(2 \alpha+1) \mathcal{D}^{\prime}$ conjugacy classes of dihedral subgroups. The lattice of conjugated subgroups for the group $D_{6}$ is presented in Fig. 2.

The theorem presented in Sec. 2.3 of the previous paper [6] immediately excludes some classes of subgroups if vectors of the considered basis are mappings, as in the case of magnetic configurations. The first excluded subgroup is the cyclic group $C_{N}$, since there is only one orbit $X=\{0,1, \ldots, N-1\}$, so $\prod_{j} \Sigma_{X_{j}}=\Sigma_{X}$ and $P\left(D_{N}\right) \cap \Sigma_{X}=P\left(D_{N}\right) \neq P\left(C_{N}\right)$. In the case of $N$ even two other subgroups are excluded: the dihedral subgroup $D_{N / 2}^{1}$ and the cyclic subgroup $C_{N / 2}$. In the first case only one orbit $X$ is again obtained, whereas $C_{N / 2}$ decomposes $X$ into subsets $X_{0}$ and $X_{1}$ containing nodes with even and odd indices, respectively. Then one obtains $P\left(D_{N}\right) \cap\left(\Sigma_{X_{0}} \times \Sigma_{X_{1}}\right)=P\left(D_{N / 2}^{0}\right) \neq P\left(C_{N / 2}\right)$. Therefore, these subgroups can be omitted in the further considerations of spin configurations. For example, when $N=6$ subgroups $C_{6}, D_{3}^{1}$, and $C_{3}$ are excluded. 


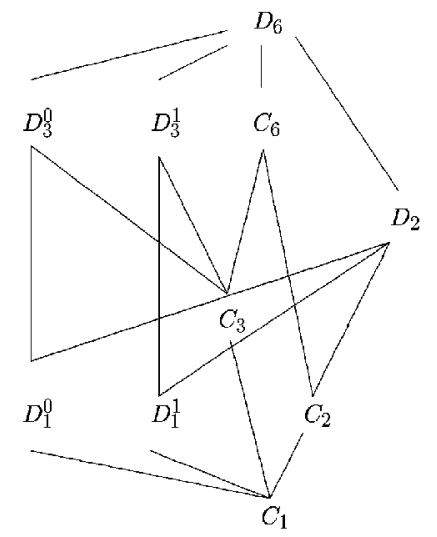

Fig. 2. Lattice of conjugated subgroups for the dihedral group $D_{6}$; lines correspond to the group-subgroup relations.

\subsection{Cosets}

For each representative $U$ of a class of conjugated subgroups $\widetilde{U}$ left coset representatives have to be chosen and fixed. The index $\left|D_{N}\right| /\left|D_{l}\right|=\lambda$ and the rotations $C_{N}^{j}, j=0,1, \ldots, \lambda-1$ will play this role. In the case of cyclic subgroups $C_{k}$ one needs additional $\lambda$ representatives. They can be chosen as $U_{j}, j=$ $0,1, \ldots, \lambda-1$. The choice of coset representatives is fixed in the further considerations.

Considering double cosets $V \backslash D_{N} / U$ we determine at first their number in different cases using results presented in Sec. 3 of the previous work [6]. If both $U$ and $V$ are cyclic then only classes containing rotations $C_{N}^{j}$ have to be taken into account and one obtains

$$
\left|C_{k} \backslash D_{N} / C_{l}\right|=2 \operatorname{gcd}(\kappa, \lambda)=2 \chi,
$$

where "ged" denotes the greatest common divisor and $\kappa=N / k, \lambda=N / l$ are co-divisors of $k$ and $l$, respectively. Note that for mutually prime co-divisors $\kappa, \lambda$ there are two double cosets only: one contains rotations $C_{N}^{j}$, whereas the second - elements $U_{j}$. If $V=D_{k}$ (or $U=D_{l}$ and $V=C_{k}$ ), then the only change is the order of one subgroup, so the result is

$$
\left|C_{k} \backslash D_{N} / D_{l}\right|=\left|D_{k} \backslash D_{N} / C_{l}\right|=\chi .
$$

If both subgroups are dihedral then the cases of odd and even $N$ have to be considered separately. Moreover, in the case of even $N$ a dihedral subgroup $D_{k}$ can be in one of three types: $D_{k}^{0}, D_{k}^{1}$ or $D_{k}$. In the first case it contains elements $U_{j}$ with even indices $j$, in the second case all $j$ 's are odd and in the last case odd and even indices $j$ are present. The final results are as follows:

- odd

$$
\left|D_{k} \backslash D_{N} / D_{l}\right|=(\chi+1) / 2
$$




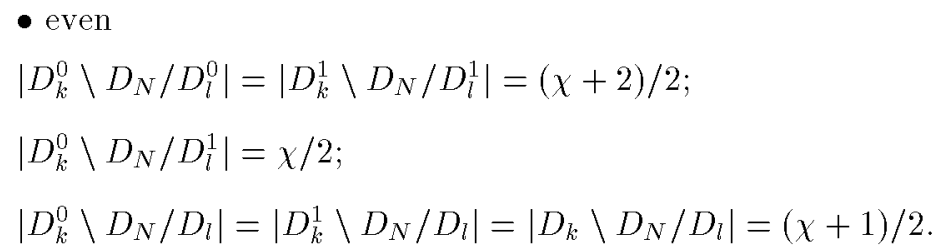

The above presented formulae are symmetric, i.e. the subgroups $U$ and $V$ can be transposed. However, in a general case a set of double cosets $V \backslash G / U$ is not symmetric, i.e. it differs from the set $U \backslash G / V$ (they have the same orders only). The same can be said about representatives $g_{d}$ of double cosets. Therefore, both cases should be considered separately and for each pair $V, U$ we obtain two sets of double coset representatives. On the other hand, the operators considered are Hermitian, so $h_{U \mu r, V \nu s}=h_{V \nu s, U \mu r}^{*}$ and, therefore, it is enough to determine one element of each pair. Nevertheless, for the sake of completeness all cases are considered below.

Since the cyclic subgroups are self-conjugated, then for a given $V$ all double cosets $V g C_{l}$ have the same order (see Eq. (13) in [6]):

$$
\left|V g C_{l}\right|=\left|C_{l} g V\right|=\frac{l|V|}{\left|V \cap C_{l}\right|} \text {. }
$$

The stabilizer in the denominator for $V=D_{k}$ or $C_{k}$ is equal to $C_{\mathrm{gcd}(k, l)}$. Therefore,

$$
\left|C_{k} g D_{l}\right|=\left|D_{k} g C_{l}\right|=2\left|C_{k} g C_{l}\right|=2 \frac{k l}{\operatorname{gcd}(k, l)}=2 \operatorname{lcm}(k, l),
$$

where "lcm" denotes the least common multiplicity. This result agrees with the above presented formulae, since $\chi=\operatorname{gcd}(\kappa, \lambda)=N / h$ with $h=\operatorname{lcm}(k, l)$. These double cosets are represented by $g_{d}=C_{N}^{j}$ with $j=0,1, \ldots, \chi-1$ if one subgroup is cyclic. If both subgroups are cyclic, then the consecutive $\chi$ representatives are $g_{d}=U_{j}, \quad 0 \leq j<\chi$. The cardinality of double cosets just obtained does not depend on the representative $g$, so Eq. (8) can be simplified if one of subgroups is cyclic. To shorten the notation let us introduce two symbols: (i) the product $B_{r s}^{U V \Gamma}\left(g_{d}\right)\left\langle U \mu g_{d}|\mathcal{H}| V \nu E\right\rangle$ is denoted as $f\left(g_{d}\right)(U, V, \Gamma$, etc. are omitted, but they can be easily determined from the context) and (ii) $\operatorname{sd}(k, l)=\operatorname{lcm}(k, l) / \operatorname{gcd}(k, l)^{\ddagger}$. Using these symbols one can write

$$
\begin{aligned}
& h_{C_{l} \mu r, C_{k} \nu s}(\Gamma)=\operatorname{sd}(k, l)^{1 / 2} \sum_{d=0}^{\chi-1} f\left(C_{N}^{d}\right)+f\left(U_{d}\right), \\
& h_{D_{l} \mu r, C_{k} \nu s}(\Gamma)=h_{C_{l} \mu r, D_{k} \nu s}(\Gamma)=[2 \operatorname{sd}(k, l)]^{1 / 2} \sum_{d=0}^{\chi-1} f\left(C_{N}^{d}\right) .
\end{aligned}
$$

\footnotetext{
‡The symbol "sd" means the symmetric division and has been introduced by analogy to the symmetric difference of sets: $\operatorname{sd}(k, l)$ is a product of primes, which appear in decomposition of either $k$ or $l$; the authors have not found any special name for this symbol in available books on the number theory.
} 
The most complicated is the case when both subgroups $U$ and $V$ are dihedral, because double cosets obtained may have different lengths and there are three different values of the number of double cosets. In fact, we are not interested in a detailed structure of double cosets, but we have to know only the number of representatives $g$ of left cosets $g U$ contained in a double coset $V g_{d} U$. The appropriate rules can be derived if one notices that the action of $V=D_{k}$ on the set of left cosets $D_{N} / D_{l}$ can be performed in two steps: (i) the action of the cyclic subgroup $C_{k} \subset D_{k}$ considered above and (ii) the action of the generator $U_{0}$ or $U_{1}$, depending on the type of $V$. The other elements $U_{q+p \kappa}, q=0,1,0<p<k$, do not yield new results since $U_{q+p \kappa}=U_{q} C_{N}^{(k-p) \kappa}$ and the action of $C_{N}^{(k-p) \kappa}$ has been already taken into account. After the first step we have at hand $\chi$ orbits represented by $C_{N}^{j}, 0 \leq j<\chi$. Each coset $C_{k} C_{N}^{j} D_{l}$ contains also $\lambda^{\prime}=\lambda / \chi$ representatives of left cosets $g D_{l}$ in the form $C_{N}^{r \chi+j}, 0 \leq r<\lambda^{\prime}$. From the multiplication rule (3) we obtain $(h=N / \chi)$ :

$$
\begin{aligned}
& U_{0} C_{N}^{j}=C_{N}^{\chi-j} U_{\chi(h-1)} \quad \text { or } \quad U_{0} C_{N}^{j}=C_{N}^{\chi-j-1} U_{\chi(h-1)+1} ; \\
& U_{1} C_{N}^{j}=C_{N}^{\chi-j+1} U_{\chi(h-1)} \quad \text { or } \quad U_{1} C_{N}^{j}=C_{N}^{\chi-j} U_{\chi(h-1)+1} .
\end{aligned}
$$

The following four cases have to be discussed:

- $U=D_{l}^{0}, V=D_{k}^{0}$ : It follows from the first formula that cosets $C_{k} C_{N}^{j} D_{l}^{0}$ and $C_{k} C_{N}^{\chi-j} D_{l}^{0}$ are merged. There is one fixed point, namely $j=0$. If $\chi$ is even then there is another fixed point $j=\chi / 2$.

- $U=D_{l}^{1}, V=D_{k}^{1}$ : In this case the last formula should be used so the results are the same as above.

- $U=D_{l}^{1}, V=D_{k}^{0}$ : Orbits $C_{k} C_{N}^{j} D_{l}^{1}$ and $C_{k} C_{N}^{j^{\prime}} D_{l}^{1}$ are merged if $j^{\prime}=-(1+j)$ $(\bmod \chi)$. The fixed point is obtained for $2 j=-1(\bmod \chi)$, so it is possible for odd $\chi$ only and in this case $j=(\chi-1) / 2$.

- $U=D_{l}^{0}, V=D_{k}^{1}$ : In this case the congruence $j^{\prime}=1-j(\bmod \chi)$ has to be satisfied to merge orbits and the fixed point is obtained for $j=(\chi+1) / 2$, if $\chi$ is odd. In this case it may be more convenient to use $j=1,2, \ldots, \chi / 2$ for $\chi$ even and $j=1,2, \ldots,(\chi-1) / 2,(\chi+1) / 2$ for $\chi$ odd.

In Table I all possible cases are presented for $N=6$ and $U, V=D_{1}^{0}, D_{1}^{1}$, and $D_{2}$. Each entry is a set of representatives $g$ of left cosets $g U$, which are contained in the same double coset $V g_{d} U$.

Taking into account the results presented above one obtains the following formulae for matrix elements:

$$
\begin{aligned}
& h_{D_{l}^{0} \mu r, D_{k}^{0} \nu s}(\Gamma)=h_{D_{l}^{1} \mu r, D_{k}^{1} \nu s}(\Gamma) \\
& \quad=\operatorname{sd}(k, l)^{1 / 2}\left(f(E)+2 \sum_{d=1}^{\chi / 2-1} f\left(C_{N}^{d}\right)+f\left(C_{N}^{\chi / 2}\right)\right)
\end{aligned}
$$


TABLE I

Representatives of cosets $g U$ contained in the same double coset $V g U$ for the dihedral group $D_{6}$ and $U$, $V=D_{1}^{0}, D_{1}^{1}, D_{2}$; underbraced elements belong to the same orbit under the action of the cyclic subgroup $C_{2}$ of $V=D_{2}$.

\begin{tabular}{c|c|c|c}
\hline \hline$U$ & $D_{1}^{0}$ & $D_{1}^{1}$ & $D_{2}$ \\
$V$ & & & \\
\hline$D_{1}^{0}$ & $\{E\}$ & $\left\{E, C_{6}^{5}\right\}$ & $\{E\}$ \\
& $\left\{C_{6}, C_{6}^{5}\right\}$ & $\left\{C_{6}, C_{6}^{4}\right\}$ & $\left\{C_{6}, C_{6}^{2}\right\}$ \\
& $\left\{C_{6}^{2}, C_{6}^{4}\right\}$ & $\left\{C_{6}^{2}, C_{6}^{3}\right\}$ & \\
& $\left\{C_{6}^{3}\right\}$ & $\{E\}$ & $\left\{E, C_{6}\right\}$ \\
\hline$D_{1}^{1}$ & $\left\{E, C_{6}\right\}$ & $\left\{C_{6}, C_{6}^{5}\right\}$ & $\left\{C_{6}^{2}\right\}$ \\
& $\left\{C_{6}^{2}, C_{6}^{5}\right\}$ & $\left\{C_{6}^{2}, C_{6}^{4}\right\}$ & \\
& $\left\{C_{6}^{3}, C_{6}^{4}\right\}$ & $\left\{C_{6}^{3}\right\}$ & \\
\hline$D_{2}$ & $\{\underbrace{\left.E, C_{6}^{3}\right\}}$ & $\{\underbrace{\left.E, C_{6}^{3}, C_{6}^{2}, C_{6}^{5}\right\}}$ & $\{E\}$ \\
& $\{\underbrace{C_{6}, C_{6}^{4}}, \underbrace{C_{6}^{2}, C_{6}^{4}}\}$ & $\left\{C_{6}^{5}\right\}$
\end{tabular}

$$
\begin{aligned}
h_{D_{l}^{\mathrm{o}} \mu r, D_{k}^{1} \nu s}(\Gamma) & =2 \operatorname{sd}(k, l)^{1 / 2} \sum_{d=1}^{\chi / 2} f\left(C_{N}^{d}\right) \\
h_{D_{l}^{\mathrm{o}} \mu r, D_{k} \nu s}(\Gamma) & =h_{D_{l} \mu r, D_{k}^{\mathrm{o} \nu s}}(\Gamma)=h_{D_{l} \mu r, D_{k} \nu s}(\Gamma) \\
=\operatorname{sd}(k, l)^{1 / 2}\left(f(E)+2 \sum_{d=1}^{(\chi-1) / 2} f\left(C_{N}^{d}\right)\right) & \\
h_{D_{l}^{1} \mu r, D_{k}^{\mathrm{o}} \nu s}(\Gamma) & =2 \operatorname{sd}(k, l)^{1 / 2} \sum_{d=0}^{\chi / 2-1} f\left(C_{N}^{d}\right) ; \\
h_{D_{l}^{1} \mu r, D_{k} \nu s}(\Gamma) & =\operatorname{sd}(k, l)^{1 / 2}\left(2 \sum_{d=0}^{(\chi-3) / 2} f\left(C_{N}^{d}\right)+f\left(C_{N}^{(\chi-1) / 2}\right)\right) \\
h_{D_{l} \mu r, D_{k}^{1} \nu s}(\Gamma) & =\operatorname{sd}(k, l)^{1 / 2}\left(2 \sum_{d=1}^{(\chi-1) / 2} f\left(C_{N}^{d}\right)+f\left(C_{N}^{(\chi+1) / 2}\right)\right) .
\end{aligned}
$$




\subsection{Transitive irreducible representations}

Let us start with the cyclic subgroups $C_{l}=\left\langle C_{N}^{\lambda}\right\rangle$ with $\lambda=N / l$. Since for all $j=0,1,2, N-1$ we have $\chi^{A_{1,2}}\left(C_{N}^{j}\right)=1$ (see Eqs. $(9,10)$, then

$$
n\left(A_{1}, R^{D_{N}: C_{l}}\right)=n\left(A_{2}, R^{D_{N}: C_{l}}\right)=1 .
$$

In the case of $N$ even there is also another pair of one-dimensional irreps, namely $B_{1}$ and $B_{2}$. It can be shown that both of them appears in the decomposition of $R^{D_{N}: C_{l}}$ if and only if $\lambda$ is even. The two-dimensional irreps $E_{p}$ always appear twice, i.e. $n\left(E_{p}, R^{D_{N}: C_{l}}\right)=2$ if $\operatorname{gcd}(p, l)=l$. Since $p<N / 2$ then only subgroups $C_{l}$ with $l<N / 2$ lead to decompositions containing $E_{p}$.

The dihedral groups $D_{l}$ have twice more elements than $C_{l}$ then the dimensions of $R^{D_{N}: D_{l}}$ are two times smaller. At first, one can notice that the irrep $A_{2}$ does not appear in the appropriate decompositions. The case $\Gamma=E_{p}$ is simple, since one obtains

$$
n\left(E_{p}, R^{D_{N}: D_{l}}\right)=n\left(E_{p}, R^{D_{N}: C_{l}}\right) / 2=1 \quad \text { if } \quad \operatorname{gcd}(p, l)=l .
$$

The irreps $B_{1}$ and $B_{2}$ (admissible for $N$ even only) appears depending on the type of the group $D_{l}$ :

$$
n\left(B_{1}, R^{D_{N}: D_{l}^{0}}\right)=n\left(B_{2}, R^{D_{N}: D_{l}^{1}}\right)=1 .
$$

The results obtained are illustrated in Table II for $N=6$.

TABLE II

Decompositions of transitive representations into irreps for $D_{6}$ (subgroups are determined by their generators).

\begin{tabular}{l|l}
\hline \hline \multicolumn{1}{c|}{$U$} & \multicolumn{1}{c}{$R^{D_{6}: U}$} \\
\hline$C_{1}=\{E\}$ & $A_{1} \oplus A_{2} \oplus B_{1} \oplus B_{2} \oplus 2 E_{1} \oplus 2 E_{2}$ \\
$D_{1}^{0}=\left\langle U_{0}\right\rangle$ & $A_{1} \oplus B_{1} \oplus E_{1} \oplus E_{2}$ \\
$D_{1}^{1}=\left\langle U_{1}\right\rangle$ & $A_{1} \oplus B_{2} \oplus E_{1} \oplus E_{2}$ \\
$C_{2}=\left\langle C_{6}^{3}\right\rangle$ & $A_{1} \oplus A_{2} \oplus 2 E_{2}$ \\
$D_{2}=\left\langle C_{6}^{3}, U_{0}\right\rangle$ & $A_{1} \oplus E_{2}$ \\
$C_{3}=\left\langle C_{6}^{2}\right\rangle$ & $A_{1} \oplus A_{2} \oplus B_{1} \oplus B_{2}$ \\
$D_{3}^{0}=\left\langle C_{6}^{2}, U_{0}\right\rangle$ & $A_{1} \oplus B_{1}$ \\
$D_{3}^{1}=\left\langle C_{6}^{2}, U_{1}\right\rangle$ & $A_{1} \oplus B_{2}$ \\
$C_{6}=\left\langle C_{6}\right\rangle$ & $A_{1} \oplus A_{2}$ \\
$D_{6}=\left\langle C_{6}, U_{0}\right\rangle$ & $A_{1}$
\end{tabular}




\subsection{Reduction coefficients}

A general formula for the reduction coefficients, see Sec. 2.4 in [6], yields that one-dimensional irreps $\Gamma$ always lead to trivial reduction coefficients $A^{U \Gamma}=1$. The indices $\gamma=1$ and $r=1$ can be omitted. So only the case $\Gamma=E_{p}$ has to be considered in more details. The vector of irreducible basis related with the reduction $E_{p} \downarrow U$ in this case can be written as

$$
\left|U E_{p} r\right\rangle=A_{x r}^{U E_{p}}\left|E_{p} x\right\rangle+A_{y r}^{U E_{p}}\left|E_{p} y\right\rangle
$$

where $r=1$ for the dihedral $\left(D_{l}\right)$ and $r=1,2$ for the cyclic $\left(C_{l}\right)$ subgroups $U$, respectively. In the first case we have to distinguish subgroups $D_{l}^{0}$ and $D_{l}^{1}$. The standard choice of the basis $\left\{\left|E_{p} x\right\rangle,\left|E_{p} y\right\rangle\right\}$ and Eq. (14) yield the matrices:

$$
E_{p}\left(U_{0}\right)=\left(\begin{array}{rr}
1 & 0 \\
0 & -1
\end{array}\right), \quad E_{p}\left(U_{1}\right)=\left(\begin{array}{rr}
\cos (2 \pi p / N) & \sin (2 \pi p / N) \\
\sin (2 \pi p / N) & -\cos (2 \pi p / N)
\end{array}\right) .
$$

Therefore, one obtains (for representations $E_{p}$ appearing in the decompositions of $R^{D: D_{l}}$, i.e. for $\left.\operatorname{gcd}(p, l)=l\right)$ :

$$
\begin{aligned}
& A_{x}^{D_{l}^{0} E_{p}}=1, \quad A_{y}^{D_{l}^{0} E_{p}}=0 ; \\
& A_{x}^{D_{l}^{1} E_{p}}=\cos (\varphi / 2), \quad A_{y}^{D_{l}^{1} E_{p}}=\sin (\varphi / 2),
\end{aligned}
$$

where $\varphi=2 \pi p / N=2 \pi p_{l} / \lambda$ with $p_{l}=p / l$. The cyclic subgroups yield $r=1,2$ and it can be shown that there are no restrictions imposed on the coefficients $A_{\gamma r}^{C_{l} E_{p}}$, as long as they determine orthonormal vectors $\left|C_{l} E_{p} 1\right\rangle,\left|C_{l} E_{p} 2\right\rangle$. The simplest choice is to put

$$
\begin{array}{ll}
A_{x 1}^{C_{l} E_{p}}=1, & A_{y 1}^{C_{l} E_{p}}=0 ; \\
A_{x 2}^{C_{l} E_{p}}=0, & A_{y 2}^{C_{l} E_{p}}=1 .
\end{array}
$$

Now we have to calculate the coefficients $B_{r s}^{U V \Gamma}\left(g_{j}\right)$ for the representatives of left cosets $g_{j} U$. Let us start from one-dimensional irreps $\Gamma=A_{1}, A_{2}, B_{1}, B_{2}$. Since the coefficients $A^{U \Gamma}=1$ and $r, s=1$, then

$$
B^{U V \Gamma}\left(g_{j}\right)=\Gamma\left(g_{j}\right)=\chi^{\Gamma}\left(g_{j}\right) .
$$

Characters of these representations are given by Eqs. (9-12). In particular, the unit irrep $A_{1}$ always yields $B^{U V A_{1}}\left(g_{j}\right)=1$.

The two-dimensional irreps $E_{p}$ appear in decompositions of $R^{D_{N}: C_{l}}$ and $R^{D_{N}: D_{l}}$ for $l<N / 2$ and $p=l p_{l}$; if $U$ or $V$ is the cyclic subgroup then $E_{p}$ appears twice. Moreover, there are two types of the dihedral subgroups for $N$ even. It leads to 16 different cases of coefficients $B_{r s}^{U V E_{p}}$, which will be presented below. We are interested in the coefficients $B_{r s}^{U V E_{p}}$ when the irrep $E_{p}$ appears in the decompositions of $R^{D_{N}: U}$ and $R^{D_{N}: V}$. Let, as in Sec. 2.3, $V=C_{k}$ or $D_{k}$ and $U=C_{l}$ or $D_{l}$. Then the index $p$ of the irrep $E_{p}$ has to satisfy both conditions: $p=p_{k} k$ and $p=p_{l} l$. Therefore, $p=\operatorname{lcm}(k, l) p^{\prime}=h p^{\prime}=N p^{\prime} / \chi$ and $\varphi=2 \pi p / N=2 \pi p^{\prime} / \chi$. 
Let subgroups $U, V$ be cyclic. Then both repetition indices $r, s=1,2$, so there are four different coefficients $B_{r s}^{U V E_{p}}\left(g_{j}\right)$. We have chosen elements $C_{N}^{j}$ and $U_{j}, j=0,1, \ldots, \lambda-1$ as representatives of the left cosets $g_{j} C_{l}$ with representation matrices

$$
E_{p}\left(C_{N}^{j}\right)=\left(\begin{array}{rr}
\cos j \varphi & -\sin j \varphi \\
\sin j \varphi & \cos j \varphi
\end{array}\right), \quad E_{p}\left(U_{j}\right)=\left(\begin{array}{rr}
\cos j \varphi & \sin j \varphi \\
\sin j \varphi & -\cos j \varphi
\end{array}\right)
$$

Taking all these relations into account one obtains

$$
\begin{aligned}
& B_{11}^{C_{l} C_{k} E_{p}}\left(C_{N}^{j}\right)=B_{22}^{C_{l} C_{k} E_{p}}\left(C_{N}^{j}\right)=B_{11}^{C_{l} C_{k} E_{p}}\left(U_{j}\right)=-B_{22}^{C_{l} C_{k} E_{p}}\left(U_{j}\right)=\cos j \varphi, \\
& B_{12}^{C_{l} C_{k} E_{p}}\left(C_{N}^{j}\right)=-B_{21}^{C_{l} C_{k} E_{p}}\left(C_{N}^{j}\right)=B_{12}^{C_{l} C_{k} E_{p}}\left(U_{j}\right)=B_{21}^{C_{l} C_{k} E_{p}}\left(U_{j}\right)=\sin j \varphi .
\end{aligned}
$$

When the subgroup $V$ is dihedral then the repetition index can be omitted, but two types of dihedral subgroups have to be considered. It leads to the following formulae:

$$
\begin{aligned}
& B_{r=1}^{C_{l} D_{k}^{0} E_{p}}\left(C_{N}^{j}\right)=B_{r=1}^{C_{l} D_{k}^{0} E_{p}}\left(U_{j}\right)=\cos j \varphi, \\
& B_{r=2}^{C_{l} D_{k}^{0} E_{p}}\left(U_{j}\right)=-B_{r=2}^{C_{l} D_{k}^{0} E_{p}}\left(C_{N}^{j}\right)=\sin j \varphi, \\
& B_{r=1}^{C_{l} D_{k}^{1} E_{p}}\left(C_{N}^{j}\right)=B_{r=1}^{C_{l} D_{k}^{1} E_{p}}\left(U_{j}\right)=\cos (j-1 / 2) \varphi, \\
& B_{r=2}^{C_{l} D_{k}^{1} E_{p}}\left(U_{j}\right)=-B_{r=2}^{C_{l} D_{k}^{1} E_{p}}\left(C_{N}^{j}\right)=\sin (j-1 / 2) \varphi .
\end{aligned}
$$

The case $U=D_{l}, V=C_{k}$ is a bit easier to discuss, since the left cosets $g_{j} D_{l}$ are represented by the elements $C_{N}^{j}$ only,

$$
\begin{aligned}
& B_{s=1}^{D_{l}^{0} C_{k} E_{p}}\left(C_{N}^{j}\right)=\cos j \varphi \\
& B_{s=2}^{D_{l}^{0} C_{k} E_{p}}\left(g_{j}\right)=\sin j \varphi \\
& B_{s=1}^{D_{l}^{1} C_{k} E_{p}}\left(C_{N}^{j}\right)=\cos (j+1 / 2) \varphi \\
& B_{s=2}^{D_{l}^{1} C_{k} E_{p}}\left(g_{j}\right)=\sin (j+1 / 2) \varphi
\end{aligned}
$$

In the last four cases both subgroups are dihedral, so $g_{j}=C_{N}^{j}$ and different types of subgroups are considered. The final results are as follows:

$$
\begin{aligned}
& B^{D_{l}^{0} D_{k}^{0} E_{p}}\left(C_{N}^{j}\right)=\cos j \varphi, \\
& B^{D_{l}^{1} D_{k}^{0} E_{p}}\left(C_{N}^{j}\right)=\cos (j+1 / 2) \varphi \\
& B^{D_{l}^{0} D_{k}^{1} E_{p}}\left(C_{N}^{j}\right)=\cos (j-1 / 2) \varphi \\
& B^{D_{l}^{1} D_{k}^{1} E_{p}}\left(C_{N}^{j}\right)=\cos j \varphi .
\end{aligned}
$$

The equation presented together with Eqs. (15-22) enable us to determine matrix elements for any operator commuting with $P(g)$. 


\section{Example}

Let us consider a ring of six spins $s=1$ with the symmetry group $D_{6}$ and the antiferromagnetic interactions of the nearest-neighbors $(J=-1)$

$$
\mathcal{H}=\sum_{j=1}^{6} s_{j} \cdot s_{j+1}, \quad 6+j \equiv j
$$

The dimension of the space of states is $(2 s+1)^{N}=729$, so it is not so large, but it is sufficient to illustrate application of the method presented in this paper.

Since in this case the magnetization is a good quantum number, then at first we consider ordered and non-ordered partitions of $N=6$ into no more than $2 s+1=$ 3 parts. There are seven ordered partitions: [600], [510], [420], [411], [330], [321], and [222]. They lead to 28 non-ordered partitions, which were presented in the previous paper [6] in Table I. On the other hand, some simple combinatorial considerations yield the dimensions of subspaces with a given magnetization $M[6,10]$. The results obtained are collected in Table III. Due to the time-reversal symmetry it is enough to consider the states with $M \geq 0$.

\section{TABLE III}

Dimensions of subspaces of a given magnetization $M \geq 0$ and non-ordered partitions related with them.

\begin{tabular}{c|c|r|c}
\hline \hline$M$ & non-ordered partitions $[k]$ & $\left|\mathcal{B}_{[k]}\right|$ & $\operatorname{dim} L_{M}$ \\
\hline 0 & {$[303],[222],[141],[060]$} & $20,90,30,1$ & 141 \\
1 & {$[213],[132],[051]$} & $60,60,6$ & 126 \\
2 & {$[204],[123],[042]$} & $15,60,15$ & 90 \\
3 & {$[114],[033]$} & 30,20 & 50 \\
4 & {$[105],[024]$} & 6,15 & 21 \\
5 & {$[015]$} & 6 & 6 \\
6 & {$[006]$} & 1 & 1
\end{tabular}

The non-ordered partitions determine orbits of the symmetric group $\Sigma_{6}$. The action of $D_{6} \subset \Sigma_{6}$ decomposes them into orbits labeled by subgroups $U \subset D_{6}$ (see Sec. 2.2 and Fig. 2). These decompositions depend only on the type of $[k]$, i.e. on the ordered partition $[\kappa]$, and are presented below (the number before a subgroup symbol $U$ denotes the multiplicity of orbits of the same type $\widetilde{U}$ ):

$$
\begin{aligned}
& {[6]: D_{6} ; \quad[51]: D_{1}^{0} ; \quad[42]: D_{2}, D_{1}^{0}, D_{1}^{1} ; \quad[33]: D_{3}^{0}, D_{1}^{0}, C_{1} ;} \\
& {[411]: D_{1}^{0}, 2 C_{1} ; \quad[321]: 2 D_{1}^{0}, 4 C_{1} ; \quad[222]: C_{2}, 3 D_{1}^{0}, 3 D_{1}^{1}, 4 C_{1} .}
\end{aligned}
$$

These results yield classification of orbits by the magnetization $M$ and the type $\widetilde{U}$ :

$$
M=6: D_{6} ; \quad M=5: D_{1}^{0} ; \quad M=4: D_{2}, 2 D_{1}^{0}, D_{1}^{1}
$$




$$
\begin{aligned}
& M=3: D_{3}^{0}, 2 D_{1}^{0}, 3 C_{1} ; \quad M=2: 2 D_{2}, 4 D_{1}^{0}, 2 D_{1}^{1}, 4 C_{1} \\
& M=1: 5 D_{1}^{0}, 8 C_{1} ; \quad M=0: D_{6}, D_{3}^{0}, C_{2}, 5 D_{1}^{0}, 3 D_{1}^{1}, 7 C_{1} .
\end{aligned}
$$

Now, taking into account the decompositions presented in Table II, we can write down the multiplicities $n\left(\Gamma, P \downarrow L_{M}\right)$ for each $M \geq 0$. The numbers presented in Table IV give us the dimension of the eigenproblem $\mathcal{H}|\psi\rangle=E|\psi\rangle$ restricted to the states with a given magnetization $M$ and the symmetry described by $\Gamma$. The largest dimension is reached for $M=0$ and $\Gamma=E_{2}$. However, when we are interested in the ground state only, then $\Gamma=A_{1}$ and the largest eigenproblem has a dimension $18 \approx 729 / 40$. We can decrease these numbers even more taking into the square of the total spin $S^{2}$. Since eigenvalues of this operator are $S(S+1)$ for $0 \leq S \leq 6$, then it is enough to find the corresponding eigenvectors and express the Hamiltonian matrix in this new basis. Table IV provides us also with dimensions $\operatorname{dim} L_{M=S, T}$ of subspaces with a given total spin $S$ and the maximum magnetization $M=S$ corresponding to an irrep $\Gamma$. It is enough to subtract adjoining rows of this table (except for the first one since $\operatorname{dim} L_{M=S=N s}=\operatorname{dim} L_{M=N s}$ ), because states with $M=S$ are these with a given $M$ except for those with $S=M+1$, which are listed in the previous row. For example, the dimensions $\operatorname{dim} L_{M=S, A_{1}}$ are $5,1,6$, $2,3,0$, and 1 for $S=0,1, \ldots, 6$, respectively. In the same way one obtains that the subspace of states with $M=S=0$ has the dimension $\operatorname{dim} L_{M=S=0}=15$ and

$$
P \downarrow L_{M=S=0}=5 A_{1} \oplus 2 B_{2} \oplus E_{1} \oplus 3 E_{2},
$$

so the dimension of the Hamiltonian eigenproblem in this case is at most five. The overall maximum is reached for $M=S=2$ and $\Gamma=E_{1}$ and equals eight, i.e. about one ninetieth of the original dimension $\operatorname{dim} L=729$.

\section{TABLE IV}

Classification of states by the magnetization $M \geq 0$ and the irrep $\Gamma$.

\begin{tabular}{c|r|r|r|r|r|r|c}
\hline \hline$M$ & $A_{1}$ & $A_{2}$ & $B_{1}$ & $B_{2}$ & $E_{1}$ & $E_{2}$ & $\operatorname{dim} L_{M}$ \\
\hline 6 & 1 & 0 & 0 & 0 & 0 & 0 & 1 \\
5 & 1 & 0 & 1 & 0 & 1 & 1 & 6 \\
4 & 4 & 0 & 2 & 1 & 4 & 3 & 21 \\
3 & 6 & 3 & 6 & 3 & 8 & 8 & 50 \\
2 & 12 & 4 & 8 & 6 & 16 & 14 & 90 \\
1 & 13 & 8 & 13 & 8 & 21 & 21 & 126 \\
0 & 18 & 8 & 13 & 10 & 22 & 24 & 141 \\
\hline Total & 92 & 38 & 73 & 46 & 122 & 118 &
\end{tabular}

Let us restrict the further considerations to the case $M=S=0$ and $\Gamma=A_{1}$. There are 18 orbits grouped into four classes labeled by the non-ordered partitions [060], [141], [222], and [303]; their representatives are presented in Fig. 3. Using 
the symbols $|U[k] \mu\rangle$, where $U$ is a stabilizer, $[k]$ - a non-ordered partition, and $\mu$ - a configuration expressed as a series $\left(m_{1}, \ldots, m_{6}\right)$ with \pm standing for \pm 1 , respectively, these representatives can be written as:

$$
\begin{array}{rlrl}
|1\rangle & =\left|D_{6}[060](0,0,0,0,0,0)\right\rangle, & & |2\rangle=\left|D_{1}^{0}[141](+, 0,0,-, 0,0)\right\rangle, \\
|3\rangle & =\left|C_{1}[141](+,-, 0,0,0,0)\right\rangle, & & |4\rangle=\left|C_{1}[141](+, 0,-, 0,0,0)\right\rangle, \\
|5\rangle & =\left|C_{2}[222](+, 0,-,+, 0,-)\right\rangle, & & |6\rangle=\left|D_{1}^{0}[222](+, 0,-,+,-, 0)\right\rangle, \\
|7\rangle & =\left|D_{1}^{0}[222](0,+,-, 0,-,+)\right\rangle, & & |8\rangle=\left|D_{1}^{0}[222](-,+, 0,-, 0,+)\right\rangle, \\
|9\rangle & =\left|D_{1}^{1}[222](+,+, 0,-,-, 0)\right\rangle, & & |10\rangle=\left|D_{1}^{1}[222](+,+,-, 0,0,-)\right\rangle, \\
|11\rangle & =\left|D_{1}^{1}[222](-,-,+, 0,0,+)\right\rangle, & |12\rangle=\left|C_{1}[222](+,+, 0,-, 0,-)\right\rangle, \\
|13\rangle & =\left|C_{1}[222](+, 0,+, 0,-,-)\right\rangle, & & |14\rangle=\left|C_{1}[222](+,-,+, 0,0,-)\right\rangle, \\
|15\rangle & =\left|C_{1}[222](+,+, 0,0,-,-)\right\rangle, & & |16\rangle=\left|D_{3}^{0}[303](+,-,+,-,+,-)\right\rangle, \\
|17\rangle & =\left|D_{1}^{0}[303](+,+,-,-,-,+)\right\rangle, & |18\rangle=\left|C_{1}[303](+,+,-,+,-,-)\right\rangle .
\end{array}
$$

Each orbit gives rise to only one state $\left|j ; A_{1}\right\rangle \equiv\left|j^{\prime}\right\rangle, j=1,2, \ldots, 18$, with symmetry $\Gamma=A_{1}$ constructed as a normalized sum of all $\left|D_{6}\right| /|U|$ states in a given orbit $O(U,[k], \mu) \equiv O_{j}$.

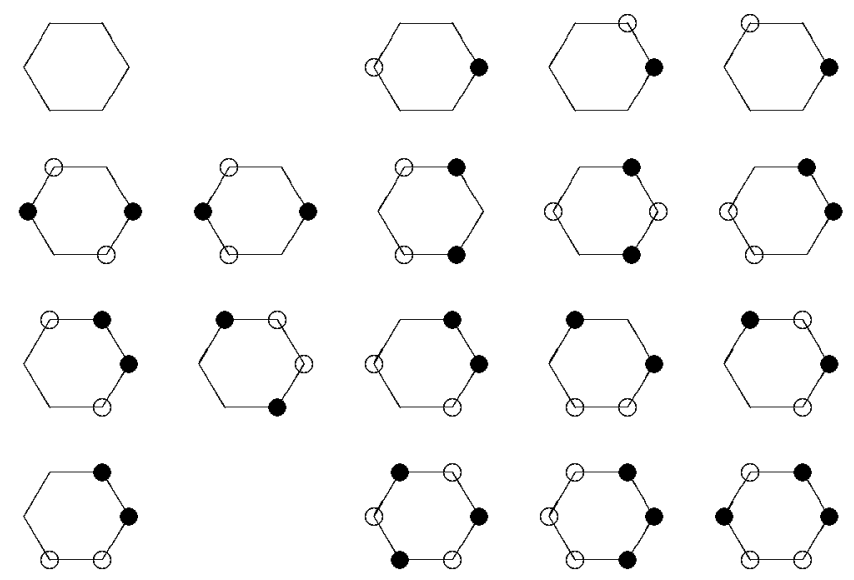

Fig. 3. Orbit representatives for $N=6, s=1$ and $M=0$; full and empty circles denote $m_{j}=+1,-1$, respectively.

Since the Hamiltonian considered is isotropic, then we can use the total spin number $S$ to label eigenstates. The operator $\boldsymbol{S}^{2}$ can be expressed as

$$
\boldsymbol{S}^{2}=\sum_{i=1}^{6} \boldsymbol{s}_{i}^{2}+2 \sum_{i=1}^{5} \sum_{i^{\prime}=i+1}^{6} \boldsymbol{s}_{i} \cdot \boldsymbol{s}_{i^{\prime}}=12+2 \mathcal{H}+2 \sum_{i=1}^{6} \boldsymbol{s}_{i} \cdot \boldsymbol{s}_{i+2}+2 \sum_{i=1}^{3} \boldsymbol{s}_{i} \cdot \boldsymbol{s}_{i+3} .
$$

The diagonal parts of $\mathcal{H}$ and $\boldsymbol{S}^{2}$ are given as

$$
\mathcal{H}_{\text {diag }}=\sum_{i=1}^{6} s_{i}^{z} s_{i+1}^{z}
$$




$$
\boldsymbol{S}_{\mathrm{diag}}^{2}=12+2 \mathcal{H}_{\mathrm{diag}}+2 \sum_{i=1}^{6} s_{i}^{z} s_{i+2}^{z}+2 \sum_{i=1}^{3} s_{i}^{z} s_{i+3}^{z} .
$$

In the first case (for $\mathcal{H}_{\text {diag }}$ ) one obtains the following 18 eigenvalues: $0,0,-1,0$, $-2,-2,-2,-2,2,-1,-1,0,0,-3,1,-6,2$, and -2 . The case of $\boldsymbol{S}^{2}$ operator is even more simple, since the diagonal values depend only on the partition $[k]$ and one obtains four numbers: 12,10 (orbits 2, 3, 4), 8 (orbits 5 through 15), and 6 (orbits 16, 17, 18).

To consider off-diagonal terms containing step operators $s_{i}^{ \pm}$we start with a graph of partitions:

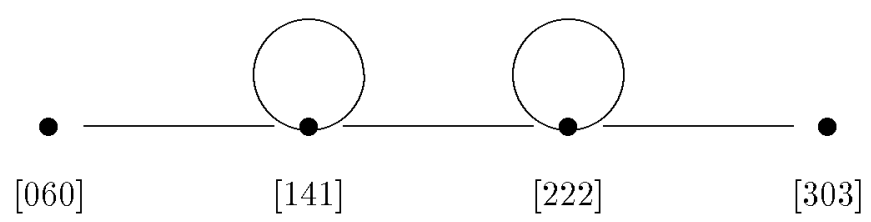

This graph leads to a block structure of off-diagonal parts of the Hamiltonian and $\boldsymbol{S}^{2}$ (symmetric) matrices:

$$
\left(\begin{array}{cccc}
\mathbf{0} & \mathcal{C} & \mathbf{0} & \mathbf{0} \\
\mathcal{C}^{\dagger} & \mathcal{A} & \mathcal{D} & \mathbf{0} \\
\mathbf{0} & \mathcal{D}^{\dagger} & \mathcal{B} & \mathcal{E} \\
\mathbf{0} & \mathbf{0} & \mathcal{E}^{\dagger} & \mathbf{0}
\end{array}\right)
$$

where rows and columns are labeled by non-ordered partitions and $\dagger$ denotes the Hermitian conjugation. All these blocks may contain non-zero elements for $\Gamma=A_{1}$, because this representation appears in the decomposition of any transitive irreps. A more detailed structure can be obtained if orbit stabilizers are taken into account (now rows and columns are labeled by pairs $(U,[k]$ ); borders of the blocks introduced above are denoted by lines):

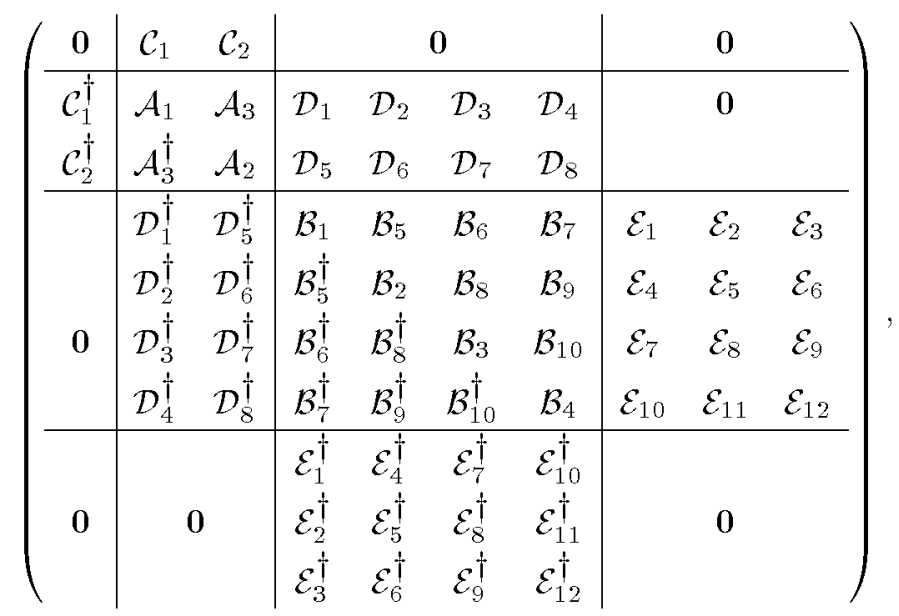


Depending on the representation considered some blocks have to contain only zeroes. For example, when $\Gamma=A_{2}$, then only blocks $\mathcal{A}_{2}, \mathcal{B}_{1}, \mathcal{B}_{4}, \mathcal{B}_{7}, \mathcal{D}_{5}, \mathcal{D}_{8}, \mathcal{E}_{3}, \mathcal{E}_{12}$ may contain non-zero entries. It follows from the fact that the irrep $A_{2}$ appears in the decomposition of a transitive representation $R^{D_{N}: U}$ if $U$ is a cyclic subgroup of $D_{N}$.

The classification of orbits with $M=0$ by type $\widetilde{U}$ yields that there are six different types of orbits, namely $D_{6}, D_{3}^{0}, C_{2}, D_{1}^{0}, D_{1}^{1}, C_{1}$, so there are 21 different double cosets to be considered. However, the matrix structure presented above excludes cosets $D_{6} g D_{6}, D_{6} g D_{3}^{0}, D_{6} g D_{1}^{1}, D_{6} g C_{2}$, and $D_{3}^{0} g D_{3}^{0}$.

Two notes are in place: (i) since we consider the case $\Gamma=A_{1}$, so all coefficients $B^{U V \Gamma}=1$; (ii) the off-diagonal terms contain products $s_{i}^{+} s_{i^{\prime}}^{-}$, which acting on kets $\left|m_{i} m_{i^{\prime}}\right\rangle$ give zero or $2\left|m_{i}+1, m_{i^{\prime}}-1\right\rangle$, therefore terms $\left(s_{i}^{+} s_{i^{\prime}}^{-}+s_{i}^{-} s_{i^{\prime}}^{+}\right) / 2$ are replaced by $\sigma_{i}^{+} \sigma_{i^{\prime}}^{-}+\sigma_{i}^{-} \sigma_{i^{\prime}}^{+}$, where $\sigma_{i}^{ \pm 1}| \pm 1\rangle=0$ and $\sigma_{i}^{ \pm}\left|m_{i}\right\rangle=\left|m_{i} \pm 1\right\rangle$, otherwise.

Let us consider, as an example, the 16th column of an operator matrix. This column corresponds to the two-element orbit containing the Néel configurations $|+,-,+,-,+,-\rangle$ and $|-,+,-,+,-,+\rangle$ with a stabilizer $D_{3}^{0}$ and labeled by the non-ordered partition [303]. Therefore, non-zero entries in this column, for operators constructed of bilinear terms, can appear only in the block $\mathcal{E}$, i.e. rows labeled by the partition [222] (except for the diagonal terms $\sum_{i, i^{\prime}} s_{i}^{z} s_{i^{\prime}}^{z}$ ). So, one has to consider four sets of double cosets $D_{3}^{0} \backslash D_{6} / U$ with $U=C_{2}, D_{1}^{0}, D_{1}^{1}$, and $C_{1}$. In all cases $k=3$ and $\kappa=3$; in the first case $\left(U=C_{2}\right) l=2, \lambda=3$, so $\operatorname{sd}(k, l)=6$ and $\chi=1$, whereas in the other cases we have $l=1, \lambda=6, \operatorname{sd}(k, l)=3$ and $\chi=2$. Equations (16), (17), and (20) yield the following formulae (the repetition indices $r, s$ may be omitted):

$$
\begin{array}{ll}
h_{C_{2} \mu, D_{3}^{0} \nu}\left(A_{1}\right)=2 \sqrt{3} f(E) ; & h_{C_{1} \mu, D_{3}^{\circ} \nu}\left(A_{1}\right)=\sqrt{6}\left(f(E)+f\left(C_{6}\right)\right) ; \\
h_{D_{1}^{1} \mu, D_{3}^{0} \nu}\left(A_{1}\right)=2 \sqrt{3} f(E) ; & h_{D_{1}^{0} \mu, D_{3}^{0} \nu}\left(A_{1}\right)=\sqrt{3}\left(f(E)+f\left(C_{6}\right)\right) .
\end{array}
$$

Therefore, one has to take into account the states $|j\rangle$, for $5 \leq j \leq 15$ and $P\left(C_{6}\right)|j\rangle$ for $j=6,7,8,12,13,14$, and 15 . Only two of them are obtained from $|16\rangle$ under the action of bilinear terms $\sigma_{i}^{+} \sigma_{i^{\prime}}^{-}$, namely

$$
P\left(C_{6}\right)|7\rangle=|+, 0,+,-, 0,-\rangle=\sigma_{2}^{+} \sigma_{5}^{-}|+,-,+,-,+,-\rangle,
$$

and

$$
|14\rangle=|+,-,+, 0,0,-\rangle=\sigma_{4}^{+} \sigma_{5}^{-}|+,-,+,-,+,-\rangle .
$$

Hence, the non-zero off-diagonal terms in the sixteenth column are

$$
\mathcal{H}_{14,16}=\sqrt{6} ; \quad \boldsymbol{S}_{7,16}^{2}=2 \sqrt{3}, \quad \boldsymbol{S}_{14,16}^{2}=2 \sqrt{6} .
$$

All other elements of operator matrices can be obtained using the following formulae:

$$
h_{C_{1} \mu, D_{6} \nu}\left(A_{1}\right)=\sqrt{2} h_{D_{1}^{0} \mu, D_{6} \nu}\left(A_{1}\right)=2 \sqrt{3} f(E),
$$




$$
\begin{aligned}
& h_{D_{1}^{\mathrm{o}} \mu, C_{2} \nu}\left(A_{1}\right)=h_{D_{1}^{1} \mu, C_{2} \nu}\left(A_{1}\right)=h_{D_{1}^{1} \mu, D_{1}^{\mathrm{o} \nu}}\left(A_{1}\right)=2 \sum_{d=0}^{2} f\left(C_{6}^{d}\right), \\
& h_{C_{1} \mu, C_{2} \nu}\left(A_{1}\right)=\sqrt{2} h_{C_{2} \mu, C_{2} \nu}\left(A_{1}\right)=\sqrt{2} \sum_{d=0}^{2}\left(f\left(C_{6}^{d}\right)+f\left(U_{d}\right)\right), \\
& h_{D_{1}^{\mathrm{o} \mu}, D_{1}^{\mathrm{o} \nu}}\left(A_{1}\right)=h_{D_{1}^{1} \mu, D_{1}^{1} \nu}\left(A_{1}\right)=f(E)+f\left(C_{6}^{3}\right)+2\left(f\left(C_{6}\right)+f\left(C_{6}^{2}\right)\right), \\
& h_{C_{1} \mu, D_{1}^{\mathrm{o} \nu} \nu}\left(A_{1}\right)=h_{C_{1} \mu, D_{1}^{1} \nu}\left(A_{1}\right)=\sqrt{2} \sum_{d=0}^{5} f\left(C_{6}^{d}\right), \\
& h_{C_{1} \mu, C_{1} \nu}\left(A_{1}\right)=\sum_{d=0}^{5}\left(f\left(C_{6}^{d}\right)+f\left(U_{d}\right)\right) .
\end{aligned}
$$

The matrices of the Hamiltonian $\mathcal{H}$ and the square of total spin $\boldsymbol{S}^{2}$ are presented in Table V.

TABLE V

The Hamiltonian (the upper triangle) and $\boldsymbol{S}^{2} / 2$ (the lower triangle) matrices for $N=6$ spins $s=1$ in the basis $\left\{\left|j^{\prime}\right\rangle \equiv\left|j ; A_{1}\right\rangle, 1 \leq j \leq 18\right\}$ (the lines are borders of blocks $\mathcal{A}, \mathcal{B}, \mathcal{C}, \mathcal{D}$, and $\mathcal{E}$ ).

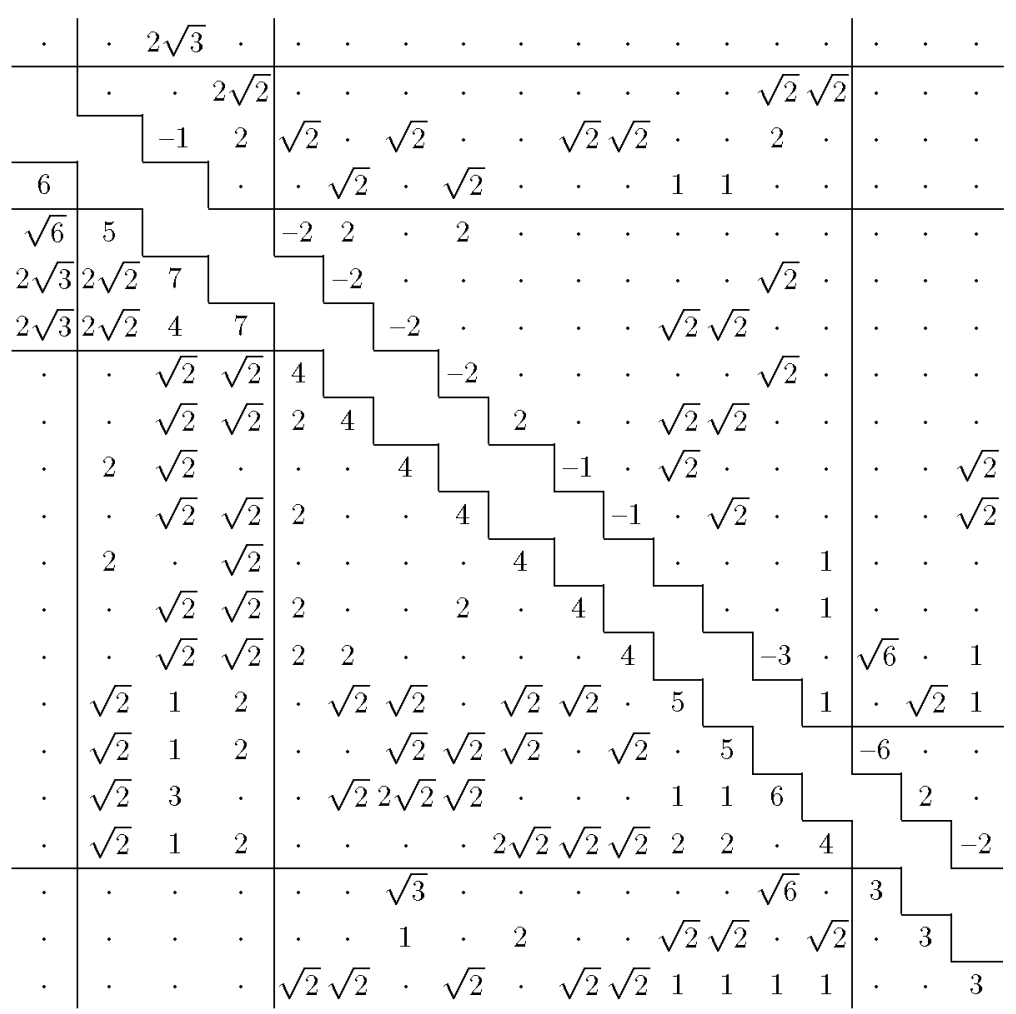


The irrep $A_{1}$ has not been chosen merely due to the simplest form of the coefficients $B^{U V A_{1}}(g)$, but in order to illustrate the other way of determination of matrix elements in this case. Let an orbit $O_{j}$ consists of $n_{j}=|G| /\left|U_{j}\right|$ elements ( $U_{j}$ is a stabilizer of the configuration $\left.|j\rangle \equiv\left|U_{j}[k] \mu\right\rangle\right)$. Hence, the fully symmetric state $\left|j^{\prime}\right\rangle \equiv\left|j ; \Gamma_{0}\right\rangle$ is given as

$$
\left|j^{\prime}\right\rangle=\frac{1}{\sqrt{n_{j}}} \sum_{i=1}^{n_{j}} P\left(g_{i}\right)|j\rangle
$$

where $g_{i}$ are representatives of left cosets $g_{i} U_{j} \in G / U_{j}$. Since a stabilizer of each element in the orbit $O_{j}$ has $\left|U_{j}\right|$ elements then

$$
\left|j^{\prime}\right\rangle=\frac{1}{\left|U_{j}\right| \sqrt{n_{j}}} \sum_{g \in G} P\left(g_{i}\right)|j\rangle=\frac{\sqrt{n_{j}}}{|G|} \sum_{g \in G} P(g)|j\rangle .
$$

Let $\mathcal{H}^{\prime}$ denote the off-diagonal part of a Hamiltonian $\mathcal{H}$, i.e. $\mathcal{H}^{\prime}=\mathcal{H}-\mathcal{H}_{\text {diag }}$. The symmetry operators $P(g)$ commute with a Hamiltonian $\mathcal{H}^{\prime}$, so

$$
\mathcal{H}^{\prime}\left|j^{\prime}\right\rangle=\frac{\sqrt{n_{j}}}{|G|} \sum_{g \in G} P(g) \mathcal{H}^{\prime}|j\rangle .
$$

To simplify the further considerations we limit ourselves to the cases $s=1 / 2,1$, when $s_{i}^{+} s_{i^{\prime}}^{-}\left|m_{i} \ldots, m_{i^{\prime}}\right\rangle=\left|m_{i}+1, m_{i^{\prime}}-1\right\rangle$ or $\sigma_{i}^{+} \sigma_{i^{\prime}}^{-}\left|m_{i}, m_{i^{\prime}} \ldots\right\rangle=\left|m_{i}+1, m_{i^{\prime}}-1\right\rangle$, since in the case of larger spin numbers a more detailed discussion is necessary. Under this assumption, each of off-diagonal terms of the bilinear Hamiltonian gives the empty state |\rangle or one configuration without any coefficients. Let $n_{j k}$ denote a number of configurations obtained from $|j\rangle$ (by the action of $\mathcal{H}^{\prime}$ ) and belonging to the orbit $O_{k}$. Then $\sum_{g} P(g) \mathcal{H}^{\prime}|j\rangle$ contains $|G| n_{j k}$ configurations from the orbit $O_{k}$. Since the state $\left|k^{\prime}\right\rangle \equiv\left|k ; A_{1}\right\rangle$ are constructed from all $n_{k}$ elements of the orbit $O_{k}$, then $|G| n_{j k}$ has to be divisible by $n_{k}$ and each element of the orbit $O_{k}$ appears $|G| n_{j k} / n_{k}$ times in the considered sum $\sum_{g} P(g) \mathcal{H}^{\prime}|j\rangle$. Therefore, one can construct $|G| n_{j k} / \sqrt{n_{k}}$ vectors $\left|k^{\prime}\right\rangle$. As the result we obtain

$$
\left\langle k^{\prime}\left|\mathcal{H}^{\prime}\right| j^{\prime}\right\rangle=\frac{\sqrt{n_{j}}}{|G|} \frac{|G| n_{j k}}{\sqrt{n_{k}}}=n_{j k} \sqrt{\frac{n_{j}}{n_{k}}}=n_{j k} \sqrt{\frac{\left|U_{k}\right|}{\left|U_{j}\right|}} .
$$

In the example presented above $|j\rangle=|+,-,+,-,+,-\rangle$ and the off-diagonal terms of the Hamiltonian give us six configurations belonging to the orbit $O_{14}$ (with $n_{14}=12$ ). The additional terms in $S^{2}$ operator lead to three configurations in the 6-element orbit $O_{7}$. Therefore,

$$
\mathcal{H}_{14,16}^{\prime}=\frac{1}{2} \boldsymbol{S}_{14,16}^{2}=6 \sqrt{\frac{2}{12}}=\sqrt{6} ; \quad \frac{1}{2} \boldsymbol{S}_{7,16}^{2}=3 \sqrt{\frac{2}{6}}=\sqrt{3},
$$

which agrees with the previous results.

The further considerations go beyond the scope of this paper, since there are some numerical rather than combinatorial problems. However, we are very close 
to the solution of the eigenproblem and it is interesting to calculate the ground state energy. In the case of isotropic Hamiltonians, when $S$ is a good quantum number, we determine an orthonormal basis for each space $L_{M=S, A_{1}}$ solving the eigenproblem $\boldsymbol{S}^{2}|\phi\rangle=S(S+1)|\phi\rangle$ for $S=0,1,2,3,4,6(S=5$ may be omitted since there are no states with $M=S=5$ and $\Gamma=A_{1}$ ). It can be done, for example, using such packages as $M A T H E M A T I C A$ or numerical libraries for calculations with an arbitrary precision as GMP [5]. Then, we transform the Hamiltonian to this new basis, which leads to a quasi-diagonal matrix consisting of blocks with dimensions 5, 1, 6, 2, 3, 1 for $S=0,1,2,3,4,6$, respectively. It is easy to solve the eigenproblems for such small blocks and, for example, in the case $S=0$ we obtain five eigenvalues: $-8.6174,-4.7979,-3.3302,-0.3391,3.0846$, where the first one corresponds to the ground state with energy per spin -1.43624. In the case of such small eigenproblems one can also determine eigenstates ${ }^{\S}$ and, therefore, it is possible to calculate spin correlations in the ground state.

\section{Final remarks}

In this paper we have studied properties of the dihedral groups $D_{N}$ to obtain analytical formulae for a number of double cosets and their elements. These results are necessary to exploit in full symmetry properties of a given spin operator and to obtain its matrix elements in the symmetry adapted basis. The groups considered are important in investigation of magnetic macromolecules forming rings or other structures with isomorphic symmetry group (e.g. crown-like structures). In a sense, this paper, together with the preceding one [6], continues works done for the cyclic groups by T. Lulek and one of the authors (W.F.) [11]. In those articles the arithmetic structure of a number of nodes $N$ was mostly taken into account and some combinatorial object were considered. The present series of papers is mainly devoted to combinatorial and group-theoretical objects and the most important result is the formula for matrix elements expressed as the sum over representatives of double cosets. In the case of dihedral groups the approach proposed has two advantages: (i) all formulae can be obtained in an analytical form and they can be used in computer programmes; (ii) in one dimension a loop over double coset representatives is, in general, shorter than a loop over all off-diagonal elements of bilinear Heisenberg Hamiltonian, which has been discussed in the previous paper [6]; moreover, it is easy to modify Hamiltonian introducing, for example, interactions with the next nearest-neighbors or alternating exchange integrals.

It should be underlined, however, that the final diagonalization of a given Hamiltonian is (almost) always done numerically, with all round-off errors caused

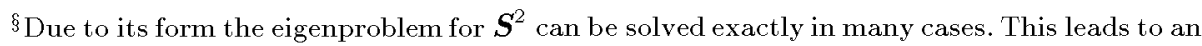
exact form of blocks in the Hamiltonian matrix. However, the Hamiltonian eigenproblem, except for some special cases, has to be solved numerically with the precision depending on software and hardware used. Therefore, all numbers obtained give only approximate values of energies, correlations, vector coefficients, etc.
} 
by the float-point representation of numbers. Secondly, the method proposed only slightly increases the size of the system which can be investigated. The so-called "combinatorial explosion" leads to very large eigenproblems for quite small systems: e.g. a system consisting of twelve spins $s=2$ yields more than 240 millions Ising configurations, so there are about ten millions orbits of the dihedral group $D_{12}$; more than 800 thousands orbits correspond to $M=0$, so this is the approximate of $\boldsymbol{S}^{2}$ eigenproblem dimension. On the other hand, a not so much smaller system with $N=8$ leads to about 2500 orbits with $M=0$, which can be investigated using modern computers. A number in the same range is obtained for $N=19$ and $s=1 / 2$, but we hope to determine the ground state energy for the Heisenberg antiferromagnetic ring with $N=24$ nodes, where a system of about 50 thousands linear equations has to be solved. However, if one would like to determine the thermodynamic properties then all states have to be taken into account, among others these with the symmetry $\Gamma=E_{p}$, which are twofold degenerate, so the dimension of $\boldsymbol{S}^{2}$ eigenproblem is approximately two times larger than that for one-dimensional irreps.

\section{Acknowledgments}

This work is partially supported by the State Committee for Scientific Research (KBN) within the project No. 2 P03B 074 19. The authors are indebted to Prof. G. Kamieniarz for fruitful discussions.

\section{References}

[1] D. Gatteschi, A. Caneschi, L. Pardi, R. Sessoli, Science 265, 1054 (1994); A. Lascialfari, D. Gatteschi, A. Cornia, U. Balucani, M.G. Pini, A. Rettori, Phys. Rev. B 57, 1115 (1998); A. Caneschi, D. Gatteschi, C. Sangregorio, R. Sessoli, L. Sorace, A. Cornia, M.A. Novak, C. Paulsen, W. Wernsdorfer, J. Magn. Magn. Mater, 200, 182 (1999); M. Affronte, J.C. Lasjaunias, A. Cornia, A. Caneschi, Phys. Rev. B 60, 1161 (1999); G. Kamieniarz, R. Matysiak, W. Florek, S. Wałcerz, J. Magn. Magn. Mater. 203, 271 (1999).

[2] W.H. Press, S.A. Teukolsky, W.T. Vetterling, B.P. Flannery, Numerical Recipes in C. The Art of Scientific Computing (second edition), Cambridge Univ. Press, Cambridge 1992, Sec. 2.0.

[3] J.C. Bonner, M.E. Fisher, Phys. Rev. A 135, 640 (1964); J. Oitmaa, D.D. Betts, Can. J. Phys. 56, 897 (1978).

[4] K. Fabricus, U. Löw, K.-H. Mütter, P. Ueberholz, Phys. Rev. B 44, 7476 (1991) and the references cited therein.

[5] More details can be found at GMP Home Pages at http://www.swox.com/gmp/.

[6] W. Florek, Acta Phys. Pol. A 100, 3 (2001).

[7] W. Florek, T. Lulek, Acta Phys. Pol. A 79, 843 (1991); W. Florek, T. Lulek, J. Phys. A, Math. Gen. 26, 2153 (1993). 
[8] H.S.M. Coxeter, W.O.J. Moser, Generators and Relations for Discrete Groups, Springer, Berlin 1957.

[9] G. Kamieniarz, R. Dekeyser, G. Musiał, L. Dębski, M. Bieliński, Phys. Rev. E 56, 144 (1997).

[10] W. Florek, Acta Magnetica II, 43 (1985); V, 145 (1988); VIII, 25 (1991).

[11] T. Lulek, J. Phys. (France) 45, 29 (1984); W. Florek, T. Lulek, J. Phys. A, Math. Gen. 20, 1921 (1987). 